\title{
Assessing the Wall Motion of Pulmonary Veins of the Left Atrium
}

\author{
WC Hu${ }^{1}$, JJ Wang $^{2}, \mathrm{HM} \mathrm{Tsao}^{3}$, LY Shyu ${ }^{1}$ \\ ${ }^{1}$ Chung Yuan Christian University, Chung Li, Taiwan, ROC \\ ${ }^{2}$ I-Shou University, Kaohsiung, Taiwan, ROC \\ ${ }^{3}$ I-Lan Hospital, I-Lan, Taiwan, ROC
}

\begin{abstract}
Atrial fibrillation is caused by some excited cardiac myocardium re-stimulated by the recurrent impulse that made the left atrium contraction irregularly. The changes of cardiac patterns could be recorded by the CT scan of the multi-slices and multi-frame technology. The abnormal rhythmic wall motion can be analyzed between the systolic of atrium and diastolic of atrium. This study has developed a $4 D$ cardiac image analysis tool to extract atrial wall motion information. Twenty set of $4 D$ $C T$ cardiac images have been tested with the developed program. The motion of interested area could be evaluated with the vector in $3 D$ with magnitude and phase angles. From the statistical analysis of motion vector over root area of four pulmonary veins, the right inferior pulmonary vein has shown abnormal motion significantly.
\end{abstract}

\section{Introduction}

This paper is to report the development of 4D cardioimages analysis program and the successful assessing atrial volume and motion of all pulmonary veins and atrium using Multi-Slice Computer Tomography (MSCT) cardiac images non-invasively. Patients with atrial fibrillation (AF) may have the deficiency of ejecting remaining blood into left ventricle after p-wave of ECG. It was hypothesized that the electrical activity arising from myocardial sleeve of pulmonary veins (PV) can trigger AF. However, the role of the sphincter function of these fibers in generation of AF remains to be elucidated.

The treatment for AF patient normally underwent ablation to block the electrical conduction pathway to minimize the extra electrical activity. The improvement of ejection fraction (EF) in left atrium can be consistent with the successful treatment of atrial ablation. To determinate the volumes change of the diastolic phase and the systolic phase of left atrium. The return of regularly atrial function can be evaluated using cardio images. [1-5] From the process and reconstruction of 4D cardiac images, the volume of atrium can be carefully estimated, if one is able to delineate the pulmonary veins and mitral valve in 3D configuration. For left atrial ejection fraction the end diastolic volume of left atrium was at the $30 \%$ time of $\mathrm{R}-\mathrm{R}$ interval. And, the end systolic volume was at the $90 \%$ time of R-R interval. Please see Figure 1 for detail illustration. Through the reconstruction and volume extraction and ejection fraction calculation process, one may also process the dimension of superior and inferior pulmonary veins. In this way, one may study if the sleeve of pulmonary veins has an important role that contributes to the atrial fibrillation. One may further study the motion of myocardium adjacent to the pulmonary veins before and after AF ablation. Thus, the more effected method of evaluation the function of left atrium non-invasively using CT cardiac images becomes important.

\section{Methods}

A self-developed program with a user friendly interface was integrated as a image processing tool for analyzing 4D cardio CT image data set. The program was developed in visual $\mathrm{C}++6.0$ that runs on window $\mathrm{XP}$ operating system. The 3D reconstruction and the atrial function analysis were able to use a minimal user interface in extracting the contour information.

The CT images were recorded in DICOM format. The cardio images data set at two distinctive time will be reading into the processing system. Then, a $3 \mathrm{D}$ cardio image will be reconstructed and displayed for inspection and further process. Two set of 3D cardio image (at ECG gated $30 \%$ time and $90 \%$ time, see Figure 1) will be registered using the rib bone as reference. The two set of images were aligned with the minimum difference of rib bone images. After the registration process, the two set of cardio images were ready for image re-sampling to extract the atrial contour. The atrial contour information will be used in the reconstruction of 3D wire mesh view display, volume calculation and wall motion. The flow 


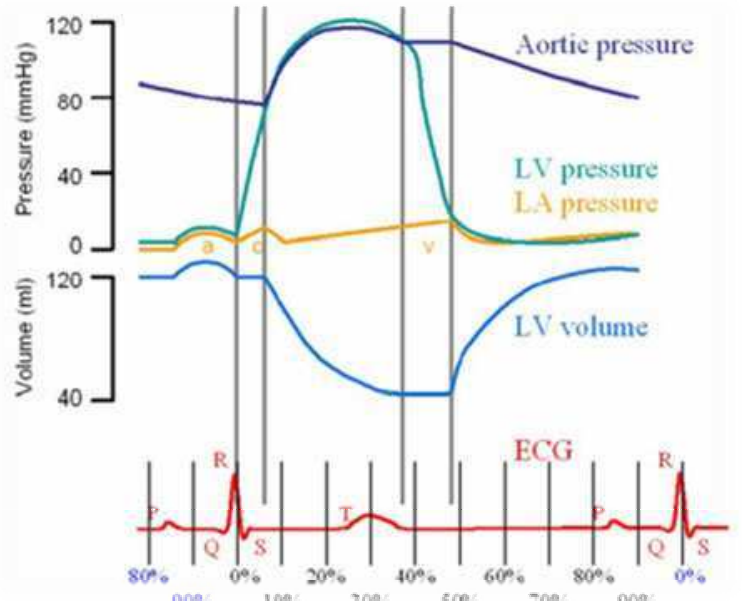

Figure 1. The R-R interval in relation to the percentage of cardiac cycle.

chart of process was illustrated in Figure 2. Twenty sets of cardio 4D images were used in this study with patients' consent.

The atrial contour was extracted using guided active contour method. [7-8] In this study, the atrial volume of two distinct time frames in a heart cycle was evaluated. One was at $30 \%$ of heart cycle which was at the end of systole of left ventricle. And, the other one was at $90 \%$ of heart cycle which was at the end of diastole of left ventricle. For each patient, there are more than 600 frames of CT gated cardio-images in one data set for processing. The processing flow chart is illustrated in Figure 2. The self-developed image analysis program will read in the data set, for example, the data set at $30 \%$ of heart cycle. And, the program will reconstruct $3 \mathrm{D}$ volume of heart. Then, using a cutting plane as reference, one may re-sample images for accurately cutting at the root of 4 pulmonary veins from the atrium and set the boundary (as shown in Figure 3 and Figure 4). Then, the user may select few slices of CT image as the seed to make a starting delineation. The self-developed heart function evaluation program will be starting to delineate the completed data set using given active contour method and seeded regional growth to delineate the atrial contour. A cubic spline procedure was performed to better fitting the atrial contour.

The method of active contour is to calculate the minimal cost function to determine the delineated contour and the edge of image. [6-7] In the case of endocardial atrial chamber, the cost function of Esnake is integrated from the weighting product of internal energy and the weighting product of external energy. The internal weighting, $w_{i}=0.3$, in this process is set to 0.3 . And, the external weighting, we, in the process is set to 0.5.
Patients with suspected AF normally underwent an ECGgated, multi-detector computed tomography (MDCT) before or after the ablation. The objective of the program is to obtain the dimension and wall motion at ostial areas of four pulmonary veins (PVs). The volume of atrium (LAV) obtained at the end-diastolic phase (ED) and endsystolic phase (ES) of left atrium (LA). The paradoxical dilatation of $\mathrm{PV}$ might indicate the poor sphincter function of PV area (PVA) [1]. The wall motion of the atrium at the root of four pulmonary veins will be calculated from the normal vector of surface between two timings of cardiac cycle. The resulting vector will be representing by the magnitude and phase. The program will calculate the dimension of the PVs area (PVA) at ED and will compare the dimension PVA at ES. The contractility of PV was defined as (PVA ES-PVA ED)/PVA ES. The atrial ejection fraction (EF) is defined as (LAV ED - LAV ES)/LAV ED. It will be calculated from the reconstructed atrial $3 \mathrm{D}$ volume representation.

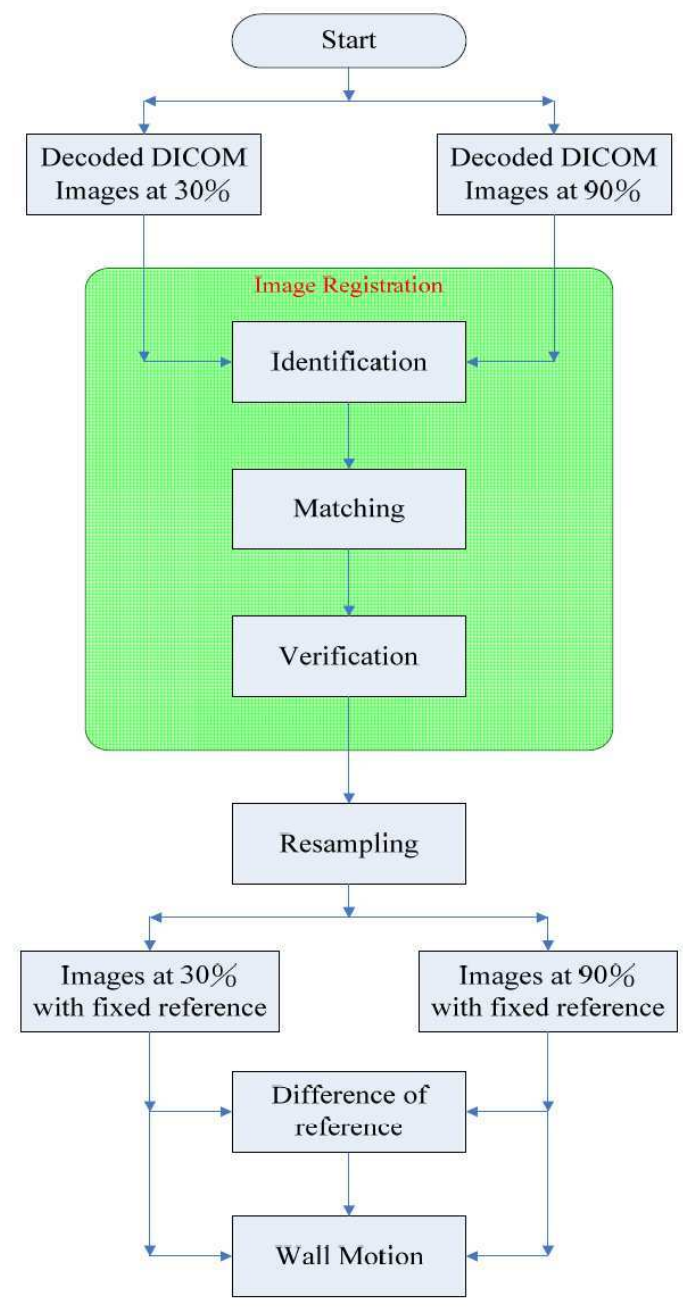

Figure 2. Processing Flow Chart 


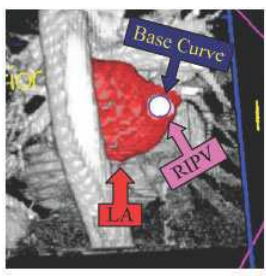

(a)

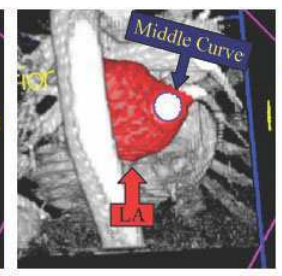

(b)

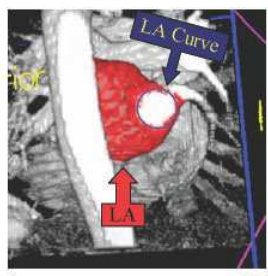

(c)
Figure 3. Cutting the plane pulmonary vein.

The red color is the atrium and white color is pulmonary vein shown with arrow. The first cut plane was shown in (a). The second cut plane was cut at $1 \mathrm{~mm}$ away from atrium (b). The third cut plane was $1 \mathrm{~mm}$ further away from second cut plane.

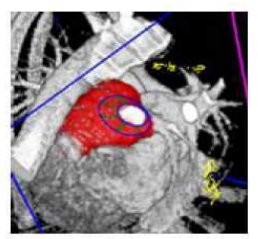

(a)

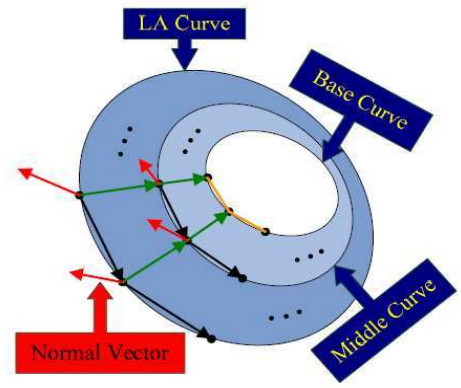

(b)
Figure 4. Illustration of the procedure for finding the motion vector at the root of pulmonary vein.

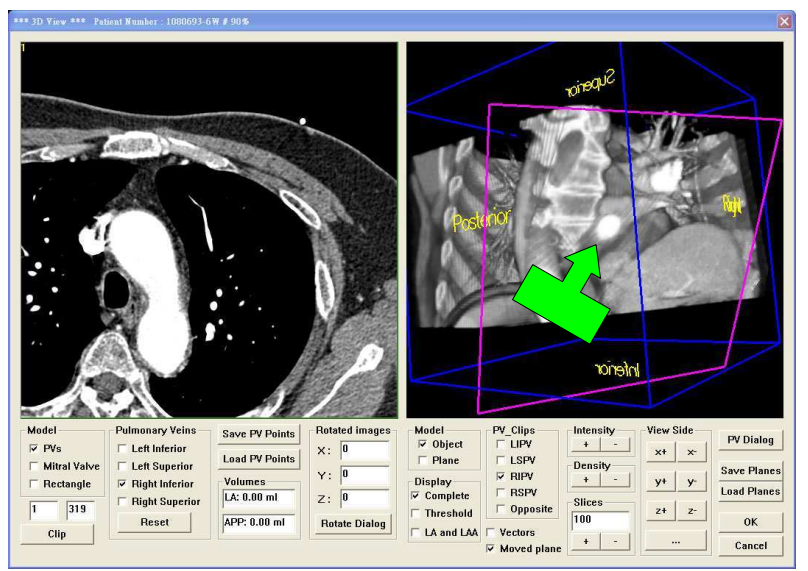

Figure 5. Illustration of the user interface and result.

The left panel shows the 2D cut plane image of purple cut plane in the right panel. The right panel is the 3D reconstruction of cardiac volume.
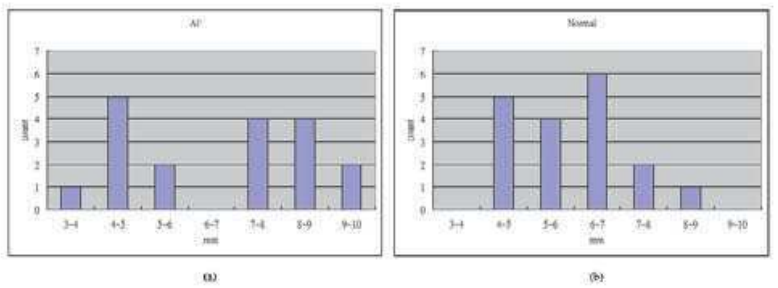

Figure 6 . The histogram of wall motion magnitude at the root of pulmonary veins.

The left panel was showing the data from AF patients. The right panel was showing the data from subject without AF symptom.

\section{Results}

The procedure of the program will be, first, reconstructing the $3 \mathrm{D}$ volume of heart. Then, a cut plane method is utilized to accurately delineate the 4 pulmonary veins and set the boundary at mitral valve for the left atrial contour extraction. The delineated information can be mapped onto the original CT images. The contour information will be re-sampled for the reconstruction of wire-mesh display. Then, the atrial volume and the dimension of pulmonary veins can be accurately assessed. The efficiency and accuracy of the developed method was reported previously. [8] The user interface and process result was illustrated as in Figure 5.

In result, twenty sets (20 patients) of MSCT cardiac images were acquired at $30 \%$ and $90 \%$ of RR interval. One set of images was excluded for its poor quality for analysis. The left atrial volume, the dimension of PV and the wall motion at root of pulmonary veins were evaluated.

This report will be concentrating on the discussion of atrial wall motion at the root of pulmonary veins. The data was divided into two groups. Group I included 11 patients (mean age $56 \pm 11$ years) with drug refractory paroxysmal AF. Group II included 8 patients (mean age $57 \pm 14$ years) without any history of AF. The wall motion of four pulmonary veins of two group's patients was examined at ED and ES. The previous result shows that the paradoxical dilatation of PV was indicating the poor sphincter function. [8] It was defined as the PV area (PVA) at ED was larger than that at ES. The wall motion at ostial areas of four PVs were obtained at the enddiastolic phase (ED) and end-systolic phase (ES) of left atrium (LA). The wall motion at the root of pulmonary vein was tallied and shown in Figure 6. For the patient without AF symptom, the distribution of movement was concentrated between 4 to $7 \mathrm{~mm}$. For the AF patient, the movement was either small $(<5 \mathrm{~mm})$ or larger than $7 \mathrm{~mm}$. 
The distribution was at two extreme of spectrum. This indicated that the abnormal wall motion of AF patient.

The wall motion at PV ostial areas except left inferior PV was significantly larger in AF patients. The higher incidence of paradoxical dilatation at ED of right superior PV $(82 \%$ versus $37 \%, \mathrm{p}<0.05)$ and left superior PV $(82 \%$ versus $43 \%, \mathrm{p}<0.05$ ) were observed in $\mathrm{AF}$ patients. In addition, poorer contractility of right superior PV was noted in AF patients ( $30 \%$ versus $1 \%, \mathrm{P}<0.05)$. Whereas, the incidence of paradoxical dilation and contractility of right inferior and left inferior PV were similar between the two groups. However, the motion vector of left inferior PV did not show significant different between two groups, whereas, the motion at the inferior and posterior quadrant of right inferior pulmonary vein for the subject without AF symptom was significantly less than AF patient. See Table 1 for reference. This result is paralleling to the previous finding.

\section{Discussion and conclusions}

This paper is to report the development of 4D cardioimages analysis system that successfully assesses atrial volume and wall motion of pulmonary veins using multiSlice CT (MSCT) cardiac images non-invasively. The procedure of the program will be, first, aligning the 3D $\mathrm{CT}$ images dataset, then, reconstructing the $3 \mathrm{D}$ volume of heart. A cut plane method is utilized to accurately delineate the 4 pulmonary veins and set the boundary at mitral valve for the left atrial contour extraction. The delineated information can be mapped onto the original CT images. The method of active contouring and seed region growth were implemented in the program for 3D atrial segmentation. The contour information will be resampled for the reconstruction of wire-mesh display. Using cut plane method, we were able to visualize and delineate intersection of the pulmonary veins. The volume of atrium was accurate estimated.

\section{Acknowledgements}

This study was supported by a grant from Nation Science Foundation, Taiwan, Republic of China (NSC-
96-2221-E-033-034-MY3 and NSC 95-2221-E-033 -034 $-\mathrm{MY} 3)$.

\section{References}

[1] Tsao HM, Wu MH, Yu WC, Tai CT, Lin YK, Hsieh MH, Ding YA, Chang MS, and Chen SA. Role of Right Middle Pulmonary Vein in Patients with Paroxysmal Atrial Fibrillation. Journal of Cardiovascular Electrophysiology, 2001; 12: 1353-1357.

[2] Tsao HM, Yu WC, Cheng HC, Wu MH, Tai CT, Lin WS, Ding YA, Chang MS, and Chen SA. Pulmonary Vein Dilation in Patients with Atrial Fibrillation: Detection by Magnetic Resonance Imaging. Journal of Cardiovascular Electrophysiology. 2001; 12 (7), 809-813.

[3] Verma A, Kilicaslan F, Adams JR, Hao S, Beheiry S, Minor S, Ozduran V, Elayi SC, Martin DO, Schweikert RA, Saliba W, Thomas JD, Garcia M, Klein A, Natale A. Extensive Ablation During Pulmonary Vein Antrum Isolation Has No Adverse Impact on Left Atrial Function: An Echocardiography and Cine Computed Tomography Analysis. J Cardiovasc Electrophysiol, 2006; 17: 741-746.

[4] Kim YH, Marom EM, Herndon JE, McAdams HP. Pulmonary Vein Diameter, Cross-sectional Area, and Shape: CT Analysis. Radiology, 2005; 235: 43-50.

[5] Hauser TH, Yeon SB, McClennen S, Katsimaglis G, Kissinger KV, Josephson ME, Rofsky NM, Manning WJ. Subclinical pulmonary vein narrowing after ablation for atrial fibrillation. Heart, 2005; 91: 672-673.

[6] Kass M, Witkin A, Terzopoulos D. Snakes: Active Contour Models. International Journal of Computer Vision. 1988; 1(4): 321-331.

[7] Wolf I, Hastenteufel M, Simone RD, Vetter M, Glombitza G, Mottl-Link S, Vahl CF, Meinzer HP. ROPES: A Semiautomated Segmentation Method for Accelerated Analysis of Three-Dimensional Echocardiographic Data. IEEE TRANSACTIONS ON MEDICAL IMAGING, 2002 21(9): 1091-1104.

[8] $\mathrm{Hu} \mathrm{WC}, \mathrm{Wu} \mathrm{MH}$, Tsao CC, Shyu LY, Wang JJ. Assessment of Left Atrial Function Using Multi-Slice CT Images. Computers in Cardiology 2007;34:85-88.

Address for correspondence

Weichih Hu, Dept. of Biomedical Engineering, Chung Yuan Christian University, Chung Li, Taiwan, ROC weichih@be.cycu.edu.tw

Table 1. Comparison of magnitude of atrial wall motion at the root of pulmonary vein.

(* indicate the significant different of two groups).

\begin{tabular}{|c|c|c|c|c|c|c|c|c|c|}
\hline & \multicolumn{4}{|c|}{ Right Inferior Pulmonary Vein } & \multicolumn{4}{|c|}{ Left Inferior Pulmonary Vein } \\
\hline & & Inferior & Anterior & Superior & Posterior & Inferior & Anterior & Superior & Posterior \\
\hline \multirow[t]{2}{*}{ AF Patient } & Average & 0.09 & 0.05 & 0.06 & 0.08 & 0.08 & 0.09 & 0.07 & 0.08 \\
\hline & SD & 0.04 & 0.02 & 0.03 & 0.03 & 0.04 & 0.04 & 0.04 & 0.05 \\
\hline \multirow[t]{2}{*}{ Patient without AF Symptom } & Average & 0.05 & 0.08 & 0.08 & 0.05 & 0.09 & 0.06 & 0.07 & 0.06 \\
\hline & SD & 0.02 & 0.07 & 0.06 & 0.02 & 0.03 & 0.04 & 0.03 & 0.04 \\
\hline \multicolumn{2}{|r|}{$\mathrm{t}$-Test ( $\mathrm{p}$ value) } & $0.01 *$ & 0.31 & 0.28 & $0.01 *$ & 0.66 & 0.11 & 0.97 & 0.38 \\
\hline
\end{tabular}

